\title{
EDITORIAL
}

\section{Referee Acknowledgements}

(C) Higher Education Press and Springer-Verlag 2011

The editors wish to thank all the referees of published articles in Vol. 5, 2010 \& Vol. 6, 2011 as well as referees who provided comments to papers they recommended rejection. We are most grateful to our referees who gave so generously of their time to provide constructive and excellent comments for authors who submitted papers to our journal.

Stephen Bahry

Limin Bai

Bao chuanyou

Mark Bray

Lorna K. S. Chan

Chen Shuangye

Chen Xiaoduan

Rebecca Clothey

Kwok-cheung Cheung

David Coniam

Michael Connelly

Hilary Cremin

Deng Meng

Ding Xiaohao

Janet Draper

Du Yuhong

Gregory P. Fairbrother

Gao Lingbiao

David Halpin

He Kekang

Vivian Heung

Hong Chengwen

W. James Jacob

Jiang Kai

Jiang Kaijun
Jin Yiming

Peggy Kong

Paula Kwan

Åse Høgsbro Lading

Wing-Wah Law

Lee Wing-on

Henry Levin

Li Jun

Li Mei

Li Qiong

Liu Jun

Liu Niancai

Liu Zhengwei

Nai Kwai Lo

Ma Yunpeng

Ma Zhenduo

Deane Neubauer

Jane Ngobia

Niu Zhikui

Pan Maoyuan

David Phillips

Kim Fong Poon-McBrayer

Suyan Pan

Glen Peterson

Filiz Polat 
Gay Garland Reed

Ren Changsong

Alan Schoenfeld

Shek Chun Ka-wai Cecilia

Shi Qiuheng

Song Zhongying

Thomas Tse

Sou Kuan Vong

Dan Wang

Wang Jiayi

Wang Ting

Wang Yingjie

Wang Yining

Anthony Welch

Margaret N. C. Wong

Wong Ping Ho
Xia Xiaohong

Xu Binyan

Xu Guoxing

Xu Shijing

Xu Yuzhen

Yan Fengqiao

Yan Guangcai

Yang Guangxue

Zhang Bing

Zhang Hua

Zhang Huajun

Zhang Jianwei

Zhao Juming

Zhou Mansheng

Zhu Zhiting 